\title{
Collision Avoidance on Shared Slots in a Wireless Slotted Network: Models and Simulations
}

\author{
Pascale Minet, Paul Muhlethaler, Ines Khoufi \\ Inria Research Center of Paris \\ 2 rue Simone Iff, 75589 Paris cedex12, France \\ Email: pascale.minet@inria.fr, paul.muhlethaler@inria.fr, ines.khoufi@inria.fr
}

\begin{abstract}
In this paper we propose an analysis of a slotted based protocol designed for devices of the Internet of Thing (IoT). In contrast to other TDMA-based protocols this scheme uses a random technique to access shared slots which presents similarities with CSMA protocols. In practice the transmissions are scheduled in a given back-off window of slots whose duration allows the transmission of a packet and its acknowledgment. Therefore this protocol can be analyzed according to the methodology introduced by Bianchi for the IEEE 802.11 protocol even if the protocol studied differs in many aspects. The model we use is also particular because we succeed in obtaining a Markov model even if the scheme used to send a packet (in a node) may depend on the transmission of the previous packet.

We distinguish two protocols; in the first one, at the initial stage or after a successful transmission, the packets are transmitted without any back-off, whereas in the second protocol each transmission is always preceded by the count down of a random back-off. Extensive simulations validate the models of both protocols and a comparative performance evaluation is carried out.
\end{abstract}

\section{INTRODUCTION}

Our purpose is to model various methods of collision avoidance on shared slots in a wireless slotted network. All the methods studied in this paper are fully distributed and based on a probabilistic transmission by each network node. More precisely, each node draws a random backoff in a backoff window. This backoff is decremented at each slot. The node waits until the backoff is zero to be allowed to transmit.

The methods vary in:

- the size of the backoff window: it may be constant or increased with the number of successive collisions.

- the reset of the backoff window: after each transmission, or only after each successful transmission, or at each first transmission of a message, etc.

- the increment of the backoff window: for instance after each unsuccessful transmission of a message, until a maximum value is reached.

Two methods will be studied and modeled, including the method used by Time Slotted Channel Hopping (TSCH) which is specified in amendment e of the IEEE 80.15.4 standard [2]. It is based on a time slotted medium access operating on several channels in parallel. Frequency hopping is used to protect against perturbations. TSCH has been designed for process automation, process control, equipment monitoring and more generally the Internet of Things. We focus on shared slots where all transmitters are allowed to transmit. These models will be validated through simulations on the NS3 simulation tool [3].

The studies [5], [6], [4], [7] analyze the slotted access of the IEEE 802.15.4 protocol and provide an evaluation of the throughput, average delay, energy consumption and reliability. All these papers propose nearly the same Markov chain model. In contrast to the present paper, the back-off evolves during the lifetime of the packet when it encounters collisions. However, when the packet is rejected (i.e; the maximum number of transmissions of this message has been reached and the last transmission collides) the protocol does not remember the actual value of the collision window for the next transmission as our model does. Moreover the models in [5], [6], [4], [7] handle the case where the node buffers always have pending packets. Our model handles the more realistic situation where we have a given packet generation rate in a single buffer with loss and provides a much simpler normalization equation than [5], [6], [4], [7].

This paper is organized as follows. In Section II we define the problem of slotted medium access to shared slots; the notation used throughout the paper is given. In Section III, we analyze the medium access protocol that always performs a backoff before each transmission. We propose a Markov model of node behavior taking into account the backoff stage and the maximum number of message transmissions. We evaluate the probabilities of success, collision and empty slots. This model is validated through simulations done with NS3. We then model the protocol that does not perform a backoff before the first transmission of each message and design its Markov model in Section IV, which is also validated by means of NS3 simulations. We compare the performance of each protocol in terms of successful message transmissions, rejected messages and node fairness in Section V. Finally, we conclude in Section VI.

\section{Problem Statement}

\section{A. Assumptions}

In this paper, we adopt the following assumptions:

- $\left.A_{1}\right)$ We consider a wireless slotted network: each transmission starts at the beginning of a slot. The slot size allows the transmission of one packet and its immediate acknowledgment when this packet is sent point-to-point.

- $\left.A_{2}\right)$ All the slots are shared. 
- $\left.A_{3}\right)$ Access to the shared slots is ruled by a backoff expressed in terms of the number of shared slots that the competing node must wait for before being allowed to transmit.

- $\left.A_{4}\right)$ There is no channel sensing before transmitting.

- $A_{5}$ ) Each network node has $N$ 1-hop neighboring nodes.

- $\left.A_{6}\right)$ Each node generates a message with a probability $1-r$.

- $\left.A_{7}\right)$ Each node has a buffer of size one. If a message is generated, when the node's buffer is not empty, it is lost.

\section{B. Notation}

We now introduce some notations that will be used in this paper. These notations are summarized in Table I.

TABLE I

NOTATIONS.

\begin{tabular}{|c|c|c|}
\hline \multicolumn{3}{|c|}{ Constants } \\
\hline Name & Meaning & Default value in TSCH \\
\hline$N$ & Number of neighboring nodes & $\in[4,32]$ \\
\hline$R$ & Maximum number of transmissions & 4 \\
\hline $\operatorname{Min} B$ & Minimum backoff stage & 1 \\
\hline $\operatorname{MaxB}$ & Maximum backoff stage & 7 \\
\hline$W_{j}$ & $\begin{array}{l}\text { Upper bound of backoff window } \\
\text { at stage } j\end{array}$ & $\begin{array}{l}W_{j}=2^{j} \\
\operatorname{Min} B \leq j \leq \operatorname{Max} B\end{array}$ \\
\hline \multicolumn{3}{|l|}{ States } \\
\hline Name & Meaning & Default value \\
\hline$(i, j, k)$ & $\begin{array}{l}\text { State of the node considered where } \\
i \text { is the number of transmissions } \\
j \text { is the backoff stage } \\
k \text { is the number of slots to wait for }\end{array}$ & $\begin{array}{l}1 \leq i \leq R \\
\operatorname{Min} B \leq j \leq \operatorname{Max} B \\
0 \leq k \leq W_{j}-1\end{array}$ \\
\hline \multicolumn{3}{|c|}{ Probabilities } \\
\hline Name & Meaning & Default value \\
\hline$p$ & $\begin{array}{l}\text { Probability of a collision when } \\
\text { a node is transmitting }\end{array}$ & \\
\hline$\tau$ & Node transmission probability & \\
\hline$r$ & Probability of staying Idle & $1-1 / \mathrm{N}$ \\
\hline
\end{tabular}

\section{Simulation}

The NS3 simulation tool is used to evaluate the performance of different methods of collision avoidance on shared slots. It also validates the models proposed.

In these simulations, different probabilities are evaluated as follows.

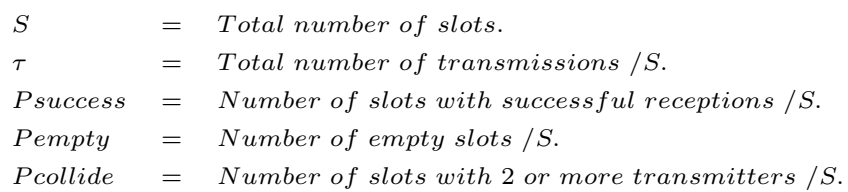

Each result is the average of 30 simulations. In each simulation, 10000 slots are simulated. The parameters given in Table I are used: the maximum number of transmissions of a message is 4 and the backoff stage belongs to the interval $[1,7]$ and $W_{j}=2^{j}$.

\section{BACKOFF BEFORE EACH TRANSMISSION}

\section{A. Principle of the method}

Each node in the slotted wireless network behaves as follows.
- Before transmitting a message, a backoff is drawn in the backoff window. The backoff window is defined by the backoff stage. The backoff stage $j$ starts with 1 and is incremented after each unsuccessful transmission (see the definition below) up to a maximum value $\operatorname{Max} B$. The backoff window at stage $j$ is equal to $\left[0, W_{j}-1\right]$. The backoff drawn in this window represents the number of shared slots the node must wait for before being allowed to transmit its message.

- When a message is sent, two cases are possible:

- either an acknowledgment is received and the transmission is considered successful. The backoff stage is reset to the initial value $\operatorname{Min} B$. The node serves the next message in its Transmit queue.

- no acknowledgment is received after a time-out and the transmission is considered unsuccessful. There are two possible cases:

* the node has already transmitted this message $R$ times, this message is rejected, a failure notification is reported to the upper layer. Notice that the backoff stage is incremented by one (up to the maximum value $\operatorname{Max} B$ ).

* otherwise, the node increments its backoff stage $j$ by one (up to the maximum value $\operatorname{Max} B$ ) and its number of transmissions for this message. It then draws a random backoff in $\left[0, W_{j}-1\right]$. When the backoff has elapsed, it transmits its message.

- The message is removed from the buffer only after its acknowledgment has been received or the maximum number of retries has been reached.

\section{B. Model based on a Markov chain}

The state of a node having an empty buffer is denoted Idle. Any message generated while the node is not in the Idle state is lost. Except in the Idle state where the buffer of the node is empty, the state of the node is represented by a triplet $(i, j, k)$ where $i$ denotes the number of transmissions of the message considered, $j$ denotes the backoff stage of the node and $k$ is the backoff drawn by the node in the backoff window $\left[0, W_{j}-1\right]$. We have the following inequalities:

- $1 \leq i \leq R$, where $R$ denotes the maximum number of transmissions of a message.

- $\operatorname{Min} B \leq j \leq \operatorname{MaxB}$, where $\operatorname{Min} B$ and $\operatorname{MaxB}$ are constant parameters fixed by the MAC protocol considered.

- $i \leq j$

- $0 \leq k \leq W_{j}-1$.

The behavior of such a node can be modeled by a Markov chain. This model, following in the footsteps of Bianchi [1] has many differences:

- There is no Clear Channel Access (CCA) before transmitting a message.

- The backoff is decremented at each shared slot even if this slot is not empty. 
- The backoff stage after $R$ unsuccessful transmissions of a message is not reset to the initial value for the next message. As a consequence, the first transmission of a new message may correspond to three different states, which we explain for the case $R=4$ and $\operatorname{Max} B=7$ :

- $(1,1,0)$, when the previous message transmission was successful. The backoff stage is equal to 1 .

- $(1,5,0)$, when the previous message was rejected. The backoff stage is equal to $R+1=5$.

- $(1,7,0)$, when the two previous messages were rejected. The backoff stage is equal to its maximum value i.e. 7 .

- Medium saturation is not assumed. We suppose that the probability of packet generation is $1-r$ at each slot.

Let us focus on the Idle state, state of a node when it has no message to transmit. We distinguish three Idle states according to the result of the previous transmission. These states are denoted:

- Idle $e_{1}$, when the previous message transmission was successful. The backoff stage will be reset to 1 for the next transmission.

- Idle $e_{2}$, when the previous message was rejected. The backoff stage will be incremented for the next transmission, it will be $R+1=5$.

- $I d l e_{3}$, when the two previous messages were rejected. The backoff stage keeps its current maximum value of 7 .

The introduction of these states allows us to model the node behavior by a Markov chain, where the next state of the node depends only on the current state and the event occurring. The Markov chain of this contention avoidance method is depicted in Figure 1, where the three Idle states are depicted by a red rectangle, whereas the three states corresponding to the first transmission of a message are depicted by a blue double-lined rectangle.

To improve the readability of the notation we denote by:

- $a_{i}$ the probability of being in state $(i, i, 0)$

for $i=1,2,3$ or $4=R$,

- $b_{i}$ the probability of being in state $(i, \min (4+i, 7), 0)$ for $i=1,2,3$ or $4=R$,

- $c_{i}$ the probability of being in state $(i, 7,0)$ for $i=1,2,3$ or $4=R$, with $\operatorname{Max} B=7<2 * R$,

- $I_{1}$ the probability of being in state $I d l e_{1}$,

- $I_{2}$ the probability of being in state $I d l e_{2}$,

- $I_{3}$ the probability of being in state $I d l e_{3}$,

- $\tau$ the probability that a node transmits in a random slot. We obtain the following system of equations:

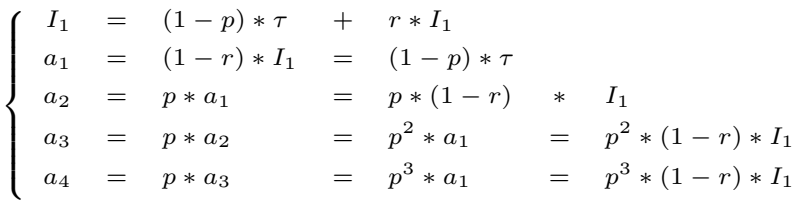

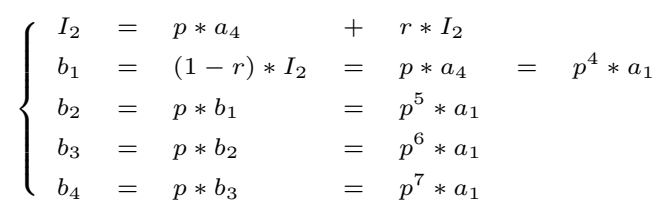

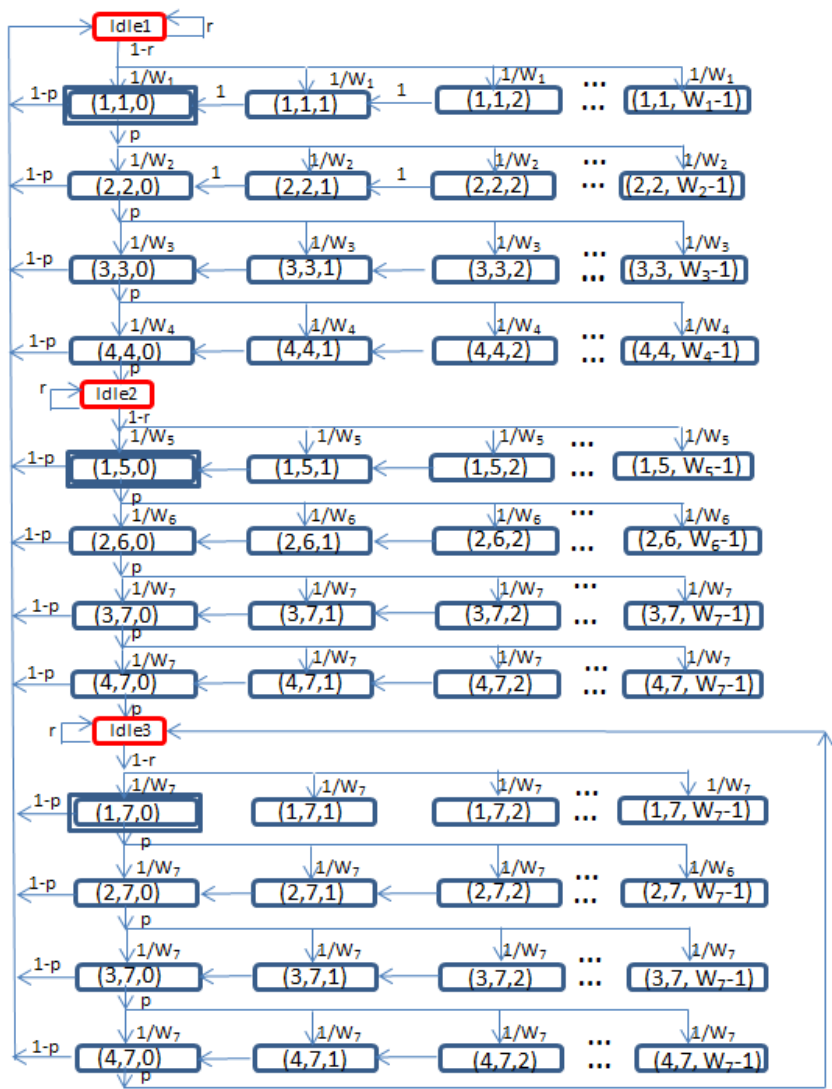

Fig. 1. The Markov chain of the collision avoidance method on shared slots with a backoff before each transmission.

$$
\begin{cases}I_{3}=p *\left(b_{4}+c 4\right)+r * I_{3} \\ c_{1}=(1-r) * I_{3} & =p *\left(b_{4}+c_{4}\right) \\ c_{2}=p * c_{1} & \\ c_{3}=p * c_{2} & =p^{2} * c_{1} \\ c_{4}=p * c_{3} & =p^{3} * c_{1} \\ c_{1}=p * b_{4} & +p * c_{4}\end{cases}
$$

We get:

$$
\begin{aligned}
& c_{1}=p * b_{4}+p^{4} * c_{1} \\
& c_{1} *\left(1-p^{4}\right)=p * b_{4}=p^{8} * a_{1} \\
& c_{1}=\frac{p^{8} * a_{1}}{1-p^{4}}
\end{aligned}
$$

If we now focus on the different backoff states before the first transmission of a message generated while in state Idle $e_{1}$, we have:

$$
\left\{\begin{aligned}
a_{1, W_{1}-1} & =a_{1} / W_{1} \\
a_{1, W_{1}-2} & =2 * a_{1} / W_{1} \\
a_{1,0} & =W_{1} * a_{1} / W_{1}
\end{aligned}\right.
$$

Summing member to member, we get:

$$
\left\{\begin{array}{l}
\sum_{k=0}^{W_{1}-1} a_{1, k}=a_{1} *\left(W_{1}+1\right) / 2 \\
\sum_{k=0}^{W_{2}-1} a_{2, k}=a_{2} *\left(W_{2}+1\right) / 2=p * a_{1} *\left(W_{2}+1\right) / 2 \\
\sum_{k=0}^{W_{3}-1} a_{3, k}=a_{3} *\left(W_{3}+1\right) / 2=p^{2} * a_{1} *\left(W_{3}+1\right) / 2 \\
\sum_{k=0}^{W_{4}-1} a_{4, k}=a_{4} *\left(W_{4}+1\right) / 2=p^{3} * a_{1} *\left(W_{4}+1\right) / 2
\end{array}\right.
$$


Similarly, we obtain:

$$
\left\{\begin{array}{l}
\sum_{k=0}^{W_{5}-1} b_{1, k}=b_{1} *\left(W_{5}+1\right) / 2=p^{4} * a_{1} *\left(W_{5}+1\right) / 2 \\
\sum_{k=0}^{W_{6}-1} b_{2, k}=b_{2} *\left(W_{6}+1\right) / 2=p^{5} * a_{1} *\left(W_{6}+1\right) / 2 \\
\sum_{k=0}^{W_{7}-1} b_{3, k}=b_{3} *\left(W_{7}+1\right) / 2=p^{6} * a_{1} *\left(W_{7}+1\right) / 2 \\
\sum_{k=0}^{W_{7}-1} b_{4, k}=b_{4} *\left(W_{7}+1\right) / 2=p^{7} * a_{1} *\left(W_{7}+1\right) / 2
\end{array}\right.
$$

and

$$
\left\{\begin{array}{l}
\sum_{k=0}^{W_{7}-1} c_{1, k}=c_{1} *\left(W_{7}+1\right) / 2=\frac{p^{8}}{1-p^{4}} * a_{1} *\left(W_{7}+1\right) / 2 \\
\sum_{k=0}^{W_{7}-1} c_{2, k}=c_{2} *\left(W_{7}+1\right) / 2=\frac{p^{9}}{1-p^{4}} * a_{1} *\left(W_{7}+1\right) / 2 \\
\sum_{k=0}^{W_{7}-1} c_{3, k}=c_{3} *\left(W_{7}+1\right) / 2=\frac{p^{10}}{1-p^{4}} * a_{1} *\left(W_{7}+1\right) / 2 \\
\sum_{k=0}^{W_{7}-1} c_{4, k}=c_{4} *\left(W_{7}+1\right) / 2=\frac{p^{11}}{1-p^{4}} * a_{1} *\left(W_{7}+1\right) / 2
\end{array}\right.
$$

For simplicity reasons, we set $A=\sum_{i=1}^{4} \sum_{k=0}^{W_{i}-1} a_{i, k}$, $B=\sum_{i=1}^{4} \sum_{k=0}^{W_{\min (i+4,7)}-1} b_{i, k}$ and $C=\sum_{i=1}^{4} \sum_{k=0}^{W_{7}-1} c_{i, k}$. We have:

$$
\begin{aligned}
& \left\{\begin{aligned}
A= & a_{1} * \frac{W_{1}+1}{2}+p * a_{1} * \frac{W_{2}+1}{2}+p^{2} * a_{1} * \frac{W_{3}+1}{2} \\
& +p^{3} * a_{1} * \frac{W_{4}+1}{2} \\
B= & p^{4} * a_{1} * \frac{W_{5}+1}{2}+p^{5} * a_{1} * \frac{W_{6}+1}{2}+p^{6} * a_{1} * \frac{W_{7}+1}{2} \\
& +p^{7} * a_{1} * \frac{W_{7}+1}{2} \\
C= & \left(1+p+p^{2}+p^{3}\right) *\left(\frac{p^{8} * a_{1}}{1-p^{4}}\right) * \frac{W_{7}+1}{2}=a_{1} * \frac{p^{8}}{1-p} * \frac{W_{7}+1}{2}
\end{aligned}\right. \\
& \left\{\begin{aligned}
A & =a_{1} *\left(\frac{W_{1}+1}{2}+p * \frac{W_{2}+1}{2}+p^{2} * \frac{W_{3}+1}{2}+p^{3} * \frac{W_{4}+1}{2}\right) \\
B & =a_{1} *\left(p^{4} * \frac{W_{5}+1}{2}+p^{5} * \frac{W_{6}+1}{2}+p^{6} * \frac{W_{7}+1}{2}+p^{7} * \frac{W_{7}+1}{2}\right) \\
C & =\frac{p^{8} * a_{1}}{1-p} * \frac{W_{7}+1}{2}
\end{aligned}\right.
\end{aligned}
$$

From the model depicted in Figure 1 and normalizing the sum of the probabilities, we get:

$$
\left\{\begin{aligned}
\tau & =a_{1}+a_{2}+a_{3}+a_{4}+b_{1}+b_{2}+b_{3}+b_{4}+c_{1}+c_{2}+c_{3}+c_{4} \\
1 & =A+B+C+I_{1}+I_{2}+I_{3} \\
I_{1} & =\frac{a_{1}}{1-r} \\
I_{2} & =\frac{p^{4} * a_{1}}{1-r} \\
I_{3} & =\frac{p^{8} * a_{1}}{(1-r) *\left(1-p^{4}\right)}
\end{aligned}\right.
$$

By replacing the $a_{i}, b_{i}$ and $c_{i}$ and $I_{i}$ by their value function of $a_{1}$ and replacing $A+B+C$ by its value computed previously, we have:

$$
\begin{aligned}
& \left\{\begin{aligned}
\tau= & a_{1} *\left(1+p+p^{2}+p^{3}+p^{4}+p^{5}+p^{6}+\frac{p^{7}}{1-p}\right) \\
1= & a_{1} *\left(\frac{W_{1}+1}{2}+p * \frac{W_{2}+1}{2}+p^{2} * \frac{W_{3}+1}{2}+p^{3} * \frac{W_{4}+1}{2}\right. \\
& +p^{4} * \frac{W_{5}+1}{2}+p^{5} * \frac{W_{6}+1}{2}+p^{6} * \frac{W_{7}+1}{2} \\
& \left.+\frac{p^{7}}{1-p} * \frac{W_{7}+1}{2}+\frac{1}{1-r}+\frac{p^{4}}{1-r}+\frac{p^{8}}{(1-r) *\left(1-p^{4}\right)}\right)
\end{aligned}\right. \\
& \left\{\begin{aligned}
\tau= & \frac{a_{1}}{1-p} \\
a_{1}= & \left(\frac{W_{1}+1}{2}+p * \frac{W_{2}+1}{2}+p^{2} * \frac{W_{3}+1}{2}+p^{3} * \frac{W_{4}+1}{2}\right. \\
& +p^{4} * \frac{W_{5}+1}{2}+p^{5} * \frac{W_{6}+1}{2}+p^{6} * \frac{W_{7}+1}{2} \\
& \left.+\frac{p^{7}}{1-p} * \frac{W_{7}+1}{2}+\frac{1}{1-r}+\frac{p^{4}}{1-r}+\frac{p^{8}}{(1-r) *\left(1-p^{4}\right)}\right)^{-1}
\end{aligned}\right.
\end{aligned}
$$

By replacing $a_{1}$ by its value as a function of $p$, we have:

$$
\begin{aligned}
\tau= & \frac{1}{1-p} \\
* \quad & \left(\frac{W_{1}+1}{2}+p * \frac{W_{2}+1}{2}+p^{2} * \frac{W_{3}+1}{2}+p^{3} * \frac{W_{4}+1}{2}+p^{4} * \frac{W_{5}+1}{2}\right. \\
& +p^{5} * \frac{W_{6}+1}{2}+p^{6} * \frac{W_{7}+1}{2}+\frac{p^{7}}{1-p} * \frac{W_{7}+1}{2}+\frac{1}{1-r}+\frac{p^{4}}{1-r} \\
& \left.+\frac{p^{8}}{(1-r) *\left(1-p^{4}\right)}\right)^{-1}
\end{aligned}
$$

We can compute $p$ the probability of a collision knowing that one node already transmits. It is equal to 1 minus the probability that none of the $N-1$ other nodes transmits. Hence, we obtain:

$$
p=1-(1-\tau)^{N-1}
$$

Replacing $p$ by its value as a function of $\tau$ in Equation 1 leads to $f(\tau)=\tau$ where the function $f($.$) is defined as$ follows:

$$
\begin{aligned}
f(\tau)= & \frac{1}{(1-\tau)^{N-1}} \\
* \quad & \left(\frac{W_{1}+1}{2}+\frac{W_{2}+1}{2} *\left(1-(1-\tau)^{N-1}\right)\right. \\
& +\frac{W_{3}+1}{2} *\left(1-(1-\tau)^{N-1}\right)^{2}+\frac{W_{4}+1}{2} *\left(1-(1-\tau)^{N-1}\right)^{3} \\
& +\frac{W_{5}+1}{2} *\left(1-(1-\tau)^{N-1}\right)^{4}+\frac{W_{6}+1}{2} *\left(1-(1-\tau)^{N-1}\right)^{5} \\
& +\frac{W_{7}+1}{2} *\left(1-(1-\tau)^{N-1}\right)^{6}+\frac{W_{7}+1}{2} * \frac{\left(1-(1-\tau)^{N-1}\right)^{7}}{(1-\tau)^{N-1}} \\
& \left.+\frac{1}{1-r}+\frac{\left(1-(1-\tau)^{N-1}\right)^{4}}{1-r}+\frac{\left(1-(1-\tau)^{N-1}\right)^{8}}{(1-r) *\left(1-\left(1-(1-\tau)^{N-1}\right)^{4}\right)}\right)^{-1}
\end{aligned}
$$

We are looking for the value of $\tau \in[0,1]$ giving $f(\tau)=\tau$. In $[0,1]$, the function $f(\tau)-\tau$ is monotonic. Hence, Equation 3 has a unique solution.

After having computed the value of $\tau$, the unique solution of Equation 3, we can compute the probabilities of success, collision and empty slots as follows.

$$
\begin{cases}a_{1} & =(1-p) * \tau \\ I_{1} & =a_{1} /(1-r)=\frac{(1-p) * \tau}{1-r} \\ I_{2} & =\frac{p^{4} * a_{1}}{1-r} \\ I_{3} & =\frac{p^{8} * a_{1}}{1-r} \\ \text { Psuccess } & =N * \tau *(1-\tau)^{N-1} \\ \text { Pempty } & =(1-\tau)^{N} \\ \text { Pcollide } & =1-\text { Psuccess }- \text { Pempty }\end{cases}
$$

\section{Model validation by simulation}

Assuming a backoff before each transmission, Figure 2 compares the results of simulations and the model for $4,8,16$ or 32 nodes, when $r=1-1 / N$. The values of Psuccess, also called normalized throughput, and Pempty are depicted. Since the value of Pcollide is deduced from the two previous, it is not depicted. Our model is validated by the very close results obtained.

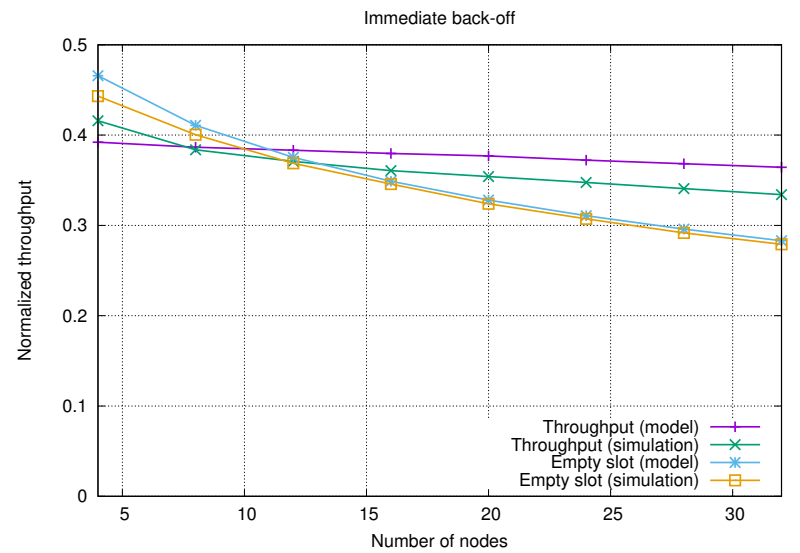

Fig. 2. Model validation. 


\section{NO BACKOFF FOR THE FIRST TRANSMISSION}

We now focus on the collision avoidance method used by $\mathrm{TSCH}$ on the shared slots. It differs from the method explained in Section III by the fact that no backoff is drawn before the first transmission of the message sent after the successful transmission of the previous message. It is also the case for the first transmission of the first message sent by the wireless node.

\section{A. Model based on a Markov chain}

The behavior of such a node can be modeled by a Markov chain. As stated above, the state of a node having an empty buffer is denoted Idle. Any message generated while the node is not in the Idle state is lost. Except in the Idle state where the buffer of the node is empty, the state of the node is represented by a triplet $(i, j, k)$ where $i$ denotes the number of transmissions of the message considered, $j$ denotes the backoff stage of the node and $k$ is the backoff drawn by the node in the backoff window $\left[0, W_{j}-1\right]$. We have the following inequalities:

- $1 \leq i \leq R$, where $R$ denotes the maximum number of transmissions of a message.

- MinB $\leq j \leq \operatorname{MaxB}$, where $\operatorname{Min} B$ and $\operatorname{MaxB}$ are constant parameters fixed by the MAC protocol considered.

- $0 \leq k \leq W_{j}-1$.

As a consequence, the first transmission of a new message may correspond to three different states, which we explain for the case $R=4$ and $\operatorname{Max} B=7$ :

- $(1,0,0)$, when the previous message transmission was successful. By convention, the backoff stage is set to 0 .

- $(1,4,0)$, when the previous message was rejected. The backoff stage is equal to $R=4$.

- $(1,7,0)$, when the two previous messages were rejected. The backoff stage is equal to its maximum value, i.e. $\operatorname{Max} B=7$.

The Markov chain of this contention avoidance method is depicted in Figure 3, where the three Idle states are depicted by a red rectangle, whereas the three states corresponding to the first transmission of a message are depicted by a blue double-lined rectangle.

To improve the readability of the notation we denote by:

- $a_{i}$ the probability of being in state $(i, i-1,0)$

for $i=1,2,3$ or $4=R$,

- $b_{i}$ the probability of being in state $(i, 3+i, 0)$ for $i=1,2,3$ or $4=R$,

- $c_{i}$ the probability of being in state $(i, 7,0)$ for $i=1,1,2,3$ or $4=R$, with $\operatorname{MaxBE}=7<2 * R$. We have the following system of equations:

$$
\left\{\begin{array}{lll}
I_{1}=(1-p) * \tau & +r * I_{1} & \\
a_{1}=(1-r) * I_{1} & =(1-p) * \tau & \\
a_{2}=p * a_{1} & =p *(1-r) * I_{1} & \\
a_{3}=p * a_{2} & =p^{2} * a_{1} & =p^{2} *(1-r) * I_{1} \\
a_{4}=p * a_{3} & =p^{3} * a_{1} & =p^{3} *(1-r) * I_{1}
\end{array}\right.
$$

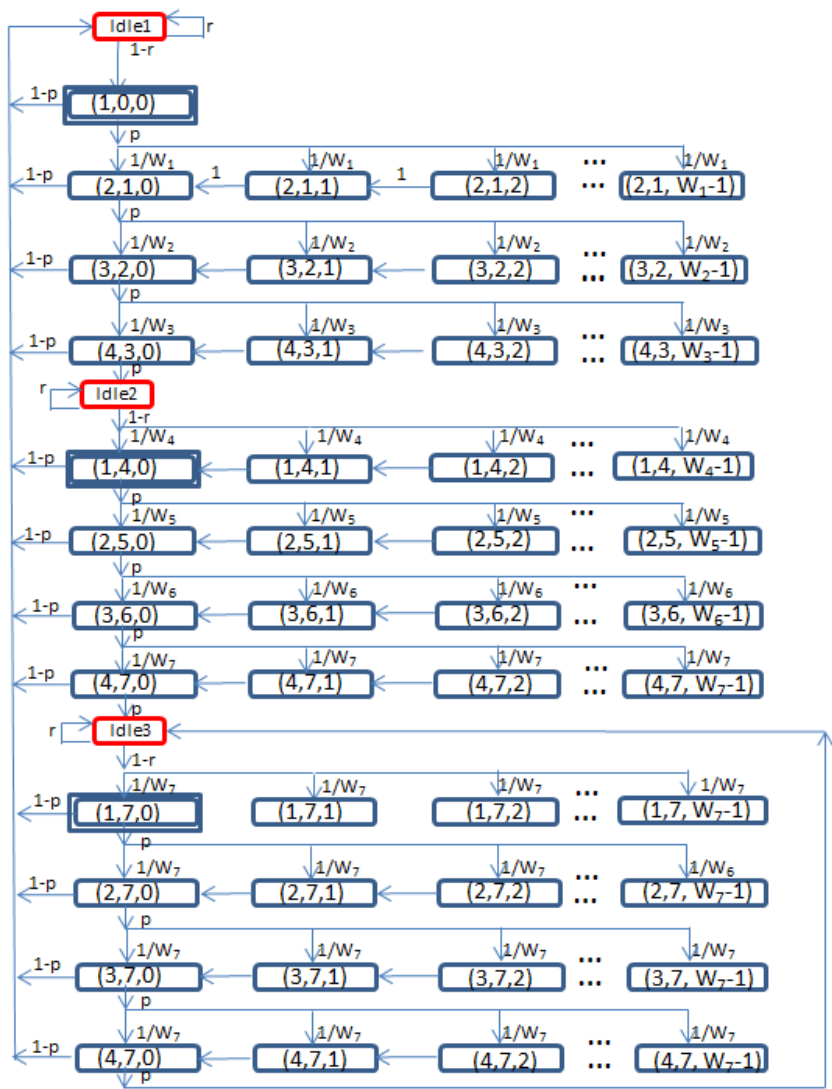

Fig. 3. The Markov chain of the collision avoidance method on shared slots of $\mathrm{TSCH}$.

$$
\begin{aligned}
& \begin{cases}I_{2}=p * a_{4} & +r * I_{2} \\
b_{1}=(1-r) * I_{2} & =p * a_{4} \\
b_{2}=p * b_{1} & =p^{5} * a_{1} \\
b_{3}=p * b_{2} & =p^{6} * a_{1} \\
b_{4}=p * b_{3} & =p^{7} * a_{1}\end{cases}
\end{aligned}
$$

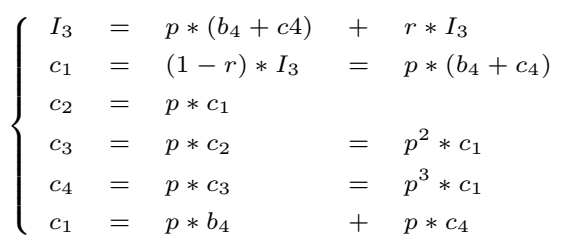

We get:

$c_{1}=\frac{p^{8} * a_{1}}{1-p^{4}}$

If now we focus on the different backoff states after the failure of the first transmission of a message generated in state $I d l e_{1}$, we obtain:

$$
\left\{\begin{array}{l}
a_{2, W_{1}-1}=a_{2} / W_{1} \\
a_{2, W_{1}-2}=2 * a_{2} / W_{1} \\
a_{2,0}=W_{1} * a_{2} / W_{1}
\end{array}\right.
$$

Summing member to member, we have:

$$
\left\{\begin{array}{l}
\sum_{k=0}^{W_{1}-1} a_{2, k}=a_{2} *\left(W_{1}+1\right) / 2=p * a_{1} *\left(W_{1}+1\right) / 2 \\
\sum_{k=0}^{W_{2}-1} a_{3, k}=a_{3} *\left(W_{2}+1\right) / 2=p^{2} * a_{1} *\left(W_{2}+1\right) / 2 \\
\sum_{k=0}^{W_{3}-1} a_{4, k}=a_{4} *\left(W_{3}+1\right) / 2=p^{3} * a_{1} *\left(W_{3}+1\right) / 2
\end{array}\right.
$$


Similarly, we get:

$$
\left\{\begin{array}{l}
\sum_{k=0}^{W_{4}-1} b_{1, k}=b_{1} *\left(W_{4}+1\right) / 2=p^{4} * a_{1} *\left(W_{4}+1\right) / 2 \\
\sum_{k=0}^{W_{5}-1} b_{2, k}=b_{2} *\left(W_{5}+1\right) / 2=p^{5} * a_{1} *\left(W_{5}+1\right) / 2 \\
\sum_{k=0}^{W_{6}-1} b_{3, k}=b_{3} *\left(W_{6}+1\right) / 2=p^{6} * a_{1} *\left(W_{6}+1\right) / 2 \\
\sum_{k=0}^{W_{7}-1} b_{4, k}=b_{4} *\left(W_{7}+1\right) / 2=p^{7} * a_{1} *\left(W_{7}+1\right) / 2
\end{array}\right.
$$

and

$\left\{\begin{array}{l}\sum_{k=0}^{W_{7}-1} c_{1, k}=c_{1} *\left(W_{7}+1\right) / 2=\frac{p^{8}}{1-p^{4}} * a_{1} *\left(W_{7}+1\right) / 2 \\ \sum_{k=0}^{W_{7}-1} c_{2, k}=c_{2} *\left(W_{7}+1\right) / 2=\frac{p^{9}}{1-p^{4}} * a_{1} *\left(W_{7}+1\right) / 2 \\ \sum_{k=0}^{W_{7}-1} c_{3, k}=c_{3} *\left(W_{7}+1\right) / 2=\frac{p^{10}}{1-p^{4}} * a_{1} *\left(W_{7}+1\right) / 2 \\ \sum_{k=0}^{W_{7}-1} c_{4, k}=c_{4} *\left(W_{7}+1\right) / 2=\frac{p^{11}}{1-p^{4}} * a_{1} *\left(W_{7}+1\right) / 2\end{array}\right.$

For simplicity reasons, we set $A=a_{1,0}+$ $\sum_{i=2}^{4} \sum_{k=0}^{W_{i-1}-1} a_{i, k}, \quad B=\sum_{i=1}^{4} \sum_{k=0}^{W_{i+3}-1} b_{i, k}$ and $C=\sum_{i=1}^{4} \sum_{k=0}^{W_{7}-1} c_{i, k}$.

$\left\{\begin{array}{l}A=a_{1} *\left(1+p * \frac{W_{1}+1}{2}+p^{2} * \frac{W_{2}+1}{2}+p^{3} * \frac{W_{3}+1}{2}\right) \\ B=a_{1} *\left(p^{4} * \frac{W_{4}+1}{2}+p^{5} * \frac{W_{5}+1}{2}+p^{6} * \frac{W_{6}+1}{2}+p^{7} * \frac{W_{7}+1}{2}\right) \\ C=\frac{p^{8} * a_{1}}{1-p} * \frac{W_{7}+1}{2}\end{array}\right.$

From the model depicted in Figure 3 and by similarity with the analysis given in Section III, we finally have:

$$
\begin{aligned}
& \left\{\begin{aligned}
\tau= & a_{1} *\left(1+p+p^{2}+p^{3}+p^{4}+p^{5}+p^{6}+\frac{p^{7}}{1-p}\right) \\
1= & a_{1} *\left(1+p * \frac{W_{1}+1}{2}+p^{2} * \frac{W_{2}+1}{2}+p^{3} * \frac{W_{3}+1}{2}\right. \\
& +p^{4} * \frac{W_{4}+1}{2}+p^{5} * \frac{W_{5}+1}{2}+p^{6} * \frac{W_{6}+1}{2} \\
& \left.+\frac{p^{7}}{1-p} * \frac{W_{7}+1}{2}+\frac{1}{1-r}+\frac{p^{4}}{1-r}+\frac{p^{8}}{(1-r) *\left(1-p^{4}\right)}\right)
\end{aligned}\right. \\
& \left\{\begin{aligned}
\tau= & \frac{a_{1}}{1-p} \\
a_{1}= & \left(1+p * \frac{W_{1}+1}{2}+p^{2} * \frac{W_{2}+1}{2}+p^{3} * \frac{W_{3}+1}{2}\right. \\
& +p^{4} * \frac{W_{4}+1}{2}+p^{5} * \frac{W_{5}+1}{2}+p^{6} * \frac{W_{6}+1}{2} \\
& \left.+\frac{p^{7}}{1-p} * \frac{W_{7}+1}{2}+\frac{1}{1-r}+\frac{p^{4}}{1-r}+\frac{p^{8}}{(1-r) *\left(1-p^{4}\right)}\right)^{-1}
\end{aligned}\right.
\end{aligned}
$$

By replacing $a_{1}$ by its value function of $p$, we obtain:

$$
\begin{aligned}
\tau= & \frac{1}{1-p} \\
* \quad & \left(1+p * \frac{W_{1}+1}{2}+p^{2} * \frac{W_{2}+1}{2}+p^{3} * \frac{W_{3}+1}{2}\right. \\
& +p^{4} * \frac{W_{4}+1}{2}+p^{5} * \frac{W_{5}+1}{2}+p^{6} * \frac{W_{6}+1}{2}+\frac{p^{7}}{1-p} * \frac{W_{7}+1}{2} \\
& \left.+\frac{1}{1-r}+\frac{p^{4}}{1-r}+\frac{p^{8}}{(1-r) *\left(1-p^{4}\right)}\right)^{-1}
\end{aligned}
$$

As in Section III, $p$ the probability of a collision knowing that one node transmits is given by Equation 2 .

Replacing $p$ by its value as a function of $\tau$ in Equation 4 leads to $f(\tau)=\tau$ where the function $f($.$) is defined as$ follows:

$$
\begin{aligned}
f(\tau)= & \frac{1}{(1-\tau)^{N-1}} \\
* \quad & \left(1+\left(1-(1-\tau)^{N-1}\right) * \frac{W_{1}+1}{2}+\left(1-(1-\tau)^{N-1}\right)^{2} * \frac{W_{2}+1}{2}\right. \\
& +\left(1-(1-\tau)^{N-1}\right)^{3} * \frac{W_{3}+1}{2}+\left(1-(1-\tau)^{N-1}\right)^{4} * \frac{W_{4}+1}{2} \\
& +\left(1-(1-\tau)^{N-1}\right)^{5} * \frac{W_{5}+1}{2}+\left(1-(1-\tau)^{N-1}\right)^{6} * \frac{W_{6}+1}{2} \\
& +\frac{\left(1-(1-\tau)^{N-1}\right)^{7}}{(1-\tau)^{N-1}} * \frac{W_{7}+1}{2}+\frac{1}{1-r} \\
& \left.+\frac{\left(1-(1-\tau)^{N-1}\right)^{4}}{1-r}+\frac{\left(1-(1-\tau)^{N-1}\right)^{8}}{(1-r) *\left(1-\left(1-(1-\tau)^{N-1}\right)^{4}\right)}\right)^{-1}
\end{aligned}
$$

We are looking for the value of $\tau \in[0,1]$ giving $f(\tau)=\tau$. In $[0,1]$, the function $f(\tau)-\tau$ is monotonic. Hence, Equation 5 has a unique solution.

After having computed the value of $\tau$, the unique solution of Equation 5, we can compute the probabilities of success, collision and empty slots as in Section III.

\section{B. Model validation by simulation}

Assuming no backoff before the first transmission, Figure 4 compares the results obtained by simulations and the model for $4,8,16$ or 32 nodes, when $r=1-1 / N$. The values of Psuccess, also called normalized throughput, and Pempty are depicted. Since the value of Pcollide is deduced from the two previous, it is not depicted. Our model is validated by the very close results obtained.

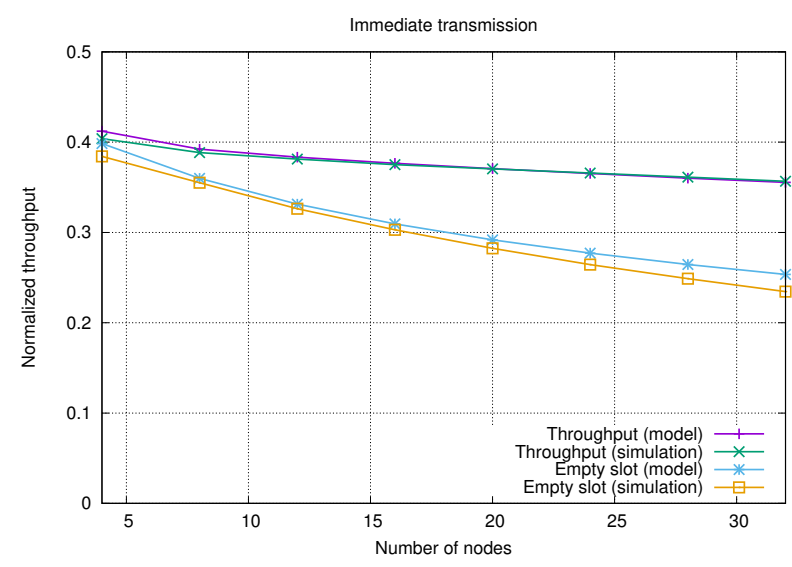

Fig. 4. Model validation.

\section{COMPARATIVE PERFORMANCE EVALUATION}

We now compare the two protocols analyzed in the two previous sections, for a number of neighboring nodes $N$ ranging from 4 to 32 . We distinguish two cases of channel load: moderate and heavy.

\section{A. Moderate medium load}

When the medium is moderately loaded, simulation results concerning the probability of success, collision and empty slots are very stable, the error bars of the simulation for a confidence interval of $95 \%$ are very small and not visible in the figures. Figure 5 shows that for a number of neighboring nodes in $[1,7]$ the protocol used by TSCH provides a higher probability of success, hence a higher normalized throughput. This is no longer the case as soon as $N \geq 8$. Similarly, simulation results show a higher number of collisions suffered by transmitting nodes for this protocol, whatever the number of nodes. This is due to the fact that the first transmission of each message very often leads to a collision. The probability of empty slots that measures the channel under-utilization is minimized by TSCH.

In this comparison, we have adopted $r=1-1 / N$ or in other words the packet generation rate is $1 / N$ for each node and in each slot. Other figures for this generation rate are acceptable and lead to a model well in accordance with simulation results. However, if the total load becomes too high, significant unfairness appears in the throughput for the TSCH protocol, as we will see in the next subsection. When the unfairness becomes high, the model is no longer able to predict the simulation results accurately. 


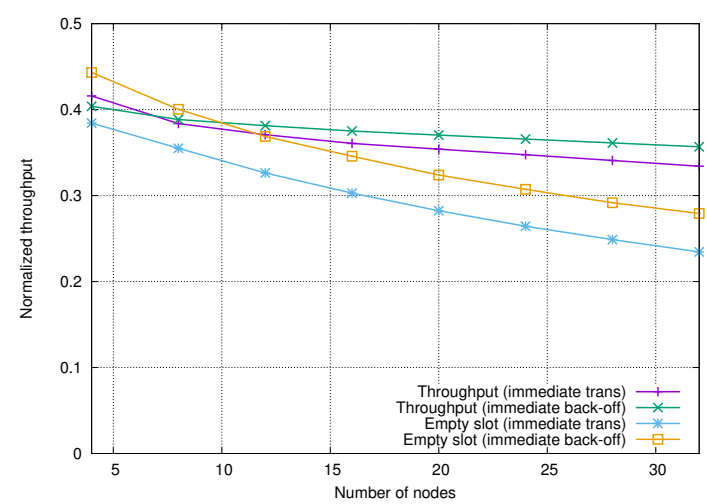

Fig. 5. Normalized throughput and probability of empty slots.

\section{B. High medium load}

When the load is high and especially in channel saturation, we are interested in the normalized throughput, the ratio of successfully transmitted messages over the total number of messages that are either successfully transmitted or rejected, as well as node fairness. Node fairness is evaluated by the Jain fairness index defined on the transmission opportunities given to a node. We have $J(T x)=\frac{\left(\sum_{i=1}^{n} T x_{i}\right)^{2}}{n \sum_{i=1}^{n} T x_{i}^{2}}$, where $T x_{i}$ denotes the number of transmission attempts of node $i$.

Figure 6 and Figure 7 allow us to compare the performance of the two collision avoidance protocols studied in this paper with the $1 / N$ Aloha protocol where each node transmits in each slot with a probability equal to $1 / N$, where $N$ is equal to 8 and 16 , respectively. In both cases, the $1 / N$ Aloha protocol provides the best node fairness (higher than 0.998) and the best ratio of successfully transmitted message (higher than 0.8525$)$. This is explained by the fact that each node receives a fair share (i.e. $1 / N$ ) of the bandwidth. The protocol used by TSCH provides the highest normalized throughput (higher than 0.4265), the smallest ratio of empty slots (less than 0.1279), but the worst fairness (less than 0.972). This is because the node that succeeds in transmitting its message, immediately transmits its next message, whereas other nodes are waiting for the end of their backoff. The protocol based on a backoff before each transmission provides a normalized throughput smaller than TSCH (0.4332 and 0.3807 for 8 and 16 nodes, respectively) but higher than the $1 / N$ Aloha protocol $(0,3927$ and 0.3806 for 8 and 16 nodes, respectively). It also ensures a better fairness than TSCH (higher than 0.986).

\section{CONCLUSION}

In this paper, we have analyzed two medium access protocols operating in a wireless slotted network. Their performances have been evaluated by means of two new Markov models that take into account the unsuccessful transmission of the previous message. Extensive simulations show a very good match between the model and the simulation results. For moderate medium load, the protocol performing a backoff before each transmission outperforms the TSCH protocol, when the number of neighboring nodes is greater than or equal to 8 . For

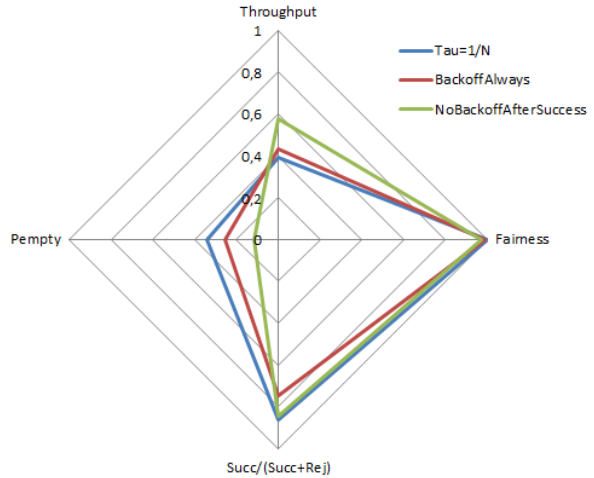

Fig. 6. Comparative performance evaluation for 8 nodes.

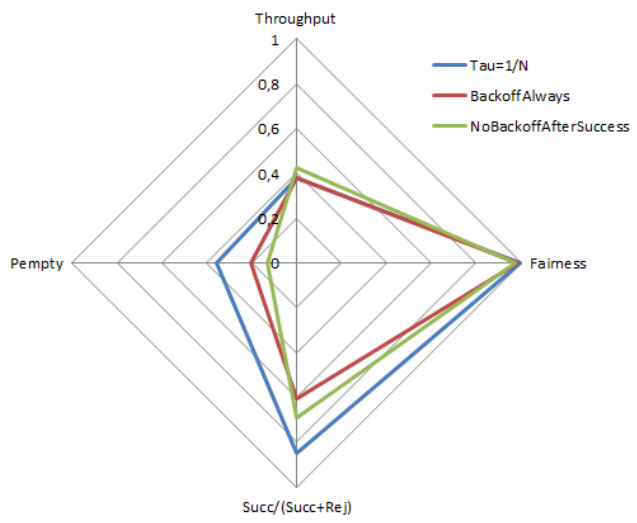

Fig. 7. Comparative performance evaluation for 16 nodes.

a smaller number of neighboring nodes, the TSCH protocol provides a higher throughput. For high medium load, the TSCH protocol provides the highest normalized throughput at the cost of some unfairness in the transmission opportunities.

\section{ACKNOWLEDGMENT}

Study co-funded by CNES and Inria in the framework of the CNES Launchers Research and Technology program.

\section{REFERENCES}

[1] G. Bianchi. Performance analysis of the IEEE 802.11 distributed coordination function. IEEE Journal on Selected Areas in Communications, 18(3):535-547, 2000.

[2] IEEE SA. IEEE Standard for Local and metropolitan area networksPart 15.4: Low-Rate Wireless Personal Area Networks (LR-WPANs) Amendment 1: MAC sublayer. IEEE Std 802.15.4e-2012 (Amendment to IEEE Std 802.15.4-2011), IEEE, February 2012.

[3] NS3-Consortium. NS3 a discrete network event simulator for internet systems.

[4] P. Park, P. Di Marco, P. Soldati, C. Fischione, and K. H. Johansson. A Generalized Markov Chain Model For Effective Analysis of Slotted IEEE 802.15.4. In Mobile Adhoc and Sensor Systems (MASS), pages 130-139. IEEE Computer Society, October 2009.

[5] T. R. Park, T. H. Kim, J. Y. Choi, Sunghyun S. Choi, and W. H. Kwon. Throughput and energy consumption analysis of IEEE 802.15. 4 slotted CSMA/CA. Electronics Letters, 41(18):1017-1019, 2005.

[6] S. Pollin, M. Ergen, S. Coleri Ergen, B. Bougard, L. Van der Perre, I. Moerman, A. Bahai, P. Varaiya, and F. Catthoor. Performance analysis of slotted carrier sense IEEE 802.15.4 medium access layer. IEEE Trans. Wireless Communications, 7(9):3359-3371, 2008.

[7] H. Wen, C. Lin, Z.-J. Chen, H. Yin, T. He, and E. Dutkiewicz. An Improved Markov Model for IEEE 802.15.4 Slotted CSMA/CA Mechanism. Journal of Computer Science and Technology, 24(3):495-504, 2009. 